\title{
ACOPLAMENTO DE CLORETO DE 4-NITROFENILDIAZÔNIO COM NUCLEÓFILOS ALIFÁTICOS: EXPERIMENTO INTEGRADO DE SÍNTESE ORGÂNICA E CRISTALOGRAFIA DE RAIOS X
}

\author{
Silvio Cunha*,\#, Monique F. Marques" e Valéria Rocha ${ }^{\#}$ \\ Instituto de Química, Universidade Federal da Bahia, Campus de Ondina, 40170-290 Salvador - BA, Brasil \\ Carlito Lariucci e Ivo Vencato \\ Instituto de Física, Universidade Federal de Goiás, CP 131, 74001-970 Goiânia - GO, Brasil
}

Recebido em 24/1/13; aceito em 2/5/13; publicado na web em 10/6/13

\begin{abstract}
AZO COUPLING OF 4-NITROPHENYLDIAZONIUM CHLORIDE WITH ALIPHATIC NUCLEOPHILES: AN INTEGRATED ORGANIC SYNTHESIS AND X-RAY CRYSTALLOGRAPHY EXPERIMENT. This article describes an undergraduate experiment for the synthesis of $p$-nitrophenyldiazonium chloride and its coupling with acetylacetone and two enaminones, 4-phenylamino-pent-3-en-2-one and 4-amino-pent-3-en-2-one, in an adaptation of a previously reported synthetic protocol. The azo dyes 4-(E)-phenylamino-3-[(E)-2-(4-nitrophenylazo)]-3-penten-2-one and 4-(E)-amino-3-[(E)-2-(4-nitrophenylazo)]-3penten-2-one were obtained, and the solid state structure of this latter azo compound was characterized by single crystal X-ray diffraction studies. This two-week integrated laboratory approach involves simple synthetic experiments and microwave chemistry in the organic laboratory plus crystallography analysis, suitable for novice students on undergraduate experimental chemistry courses.
\end{abstract}

Keywords: enaminone; azo dye; solid state structure.

\section{INTRODUÇÃO}

O acoplamento azo de cloretos de arildiazônio com anilinas ou fenóis é executado nos cursos experimentais de química orgânica como exemplo da síntese de corantes orgânicos através da formação de ligação $\mathrm{C}_{\mathrm{sp}}{ }^{2} \mathrm{~N}_{\mathrm{sp}}{ }^{2}$, Esquema $1 .^{1-5} \mathrm{O}$ emprego exclusivo de nucleófilos aromáticos nestes experimentos ilustra a potencialidade sintética dos sais de arildiazônio, mas estes sais têm aplicação bem mais ampla, como na reação de Heck-Matsuda ${ }^{6}$, na qual uma ligação $\mathrm{C}_{\mathrm{sp}}{ }^{2}-\mathrm{C}_{\mathrm{sp}}{ }^{2}$ é formada, e na reação de Sandmeyer, na qual haletos de arila ou aril-nitrilas são obtidos, ${ }^{2}$ Esquema 1 . Mesmo na reação de acoplamento azo, a reatividade dos sais de arildiazônio e a natureza dos nucleófilos que podem ser empregados são bem mais amplas que as descritas nos procedimentos disponíveis em diversos livros textos experimentais de química orgânica, ${ }^{1-5}$ o que pode induzir o estudante a uma visão limitada da aplicação sintética e dos tipos de corantes preparáveis. ${ }^{4}$

Em função do cenário acima e da nossa continuada formulação de experimentos para a graduação ao longo da última década, em consonância com as demandas modernas da formação de profissionais da química, ${ }^{7-13}$ apresentamos aqui experimentos de acoplamento azo nos quais enaminonas e acetilacetona são empregados como nucleófilos alifáticos. Duas premissas norteiam o trabalho aqui descrito; a primeira é que o graduando possa incorporar um leque mais amplo de conhecimento sobre a química dos sais de diazônio, dos compostos beta-dicarbonílicos e seus derivados. A segunda premissa, em adição aos aspectos sintéticos, é a inadiável necessidade de aproximar os experimentos de graduação da fronteira do conhecimento contemporâneo. Neste sentido, a estrutura microcristalina de um dos corantes é reinvestigada empregando-se difração de raios $\mathrm{X}$, proporcionando ao estudante de graduação formação interdisciplinar e mais próxima da realidade da pesquisa, onde a

*e-mail: silviodc@ufba.br

\#Instituto Nacional de Ciência e Tecnologia - INCT em Energia e Ambiente, Universidade Federal da Bahia, Salvador, BA

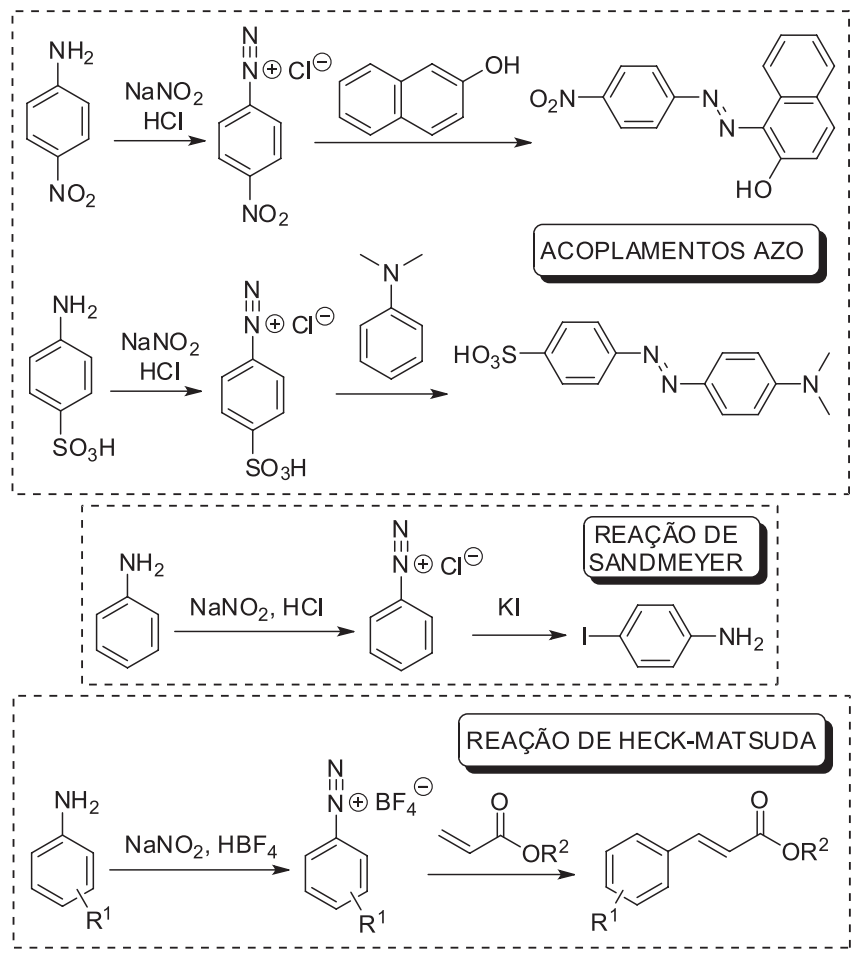

Esquema 1. Exemplos de aplicações sintéticas de sais de arildiazônio

cristalografia e a síntese química são rotineiramente associadas.

No ano em que se comemora o centenário da cristalografia de raios X como técnica de elucidação estrutural, ${ }^{14}$ e que comprovou experimentalmente a geometria tetraédrica para o átomo de carbono tetracoordenado, ${ }^{15}$ os experimentos aqui elaborados proporcionam ao aluno conhecer esta poderosa técnica de determinação estrutural e a valiosa ciência cristalografia de raios X, geralmente negligenciada nos cursos de graduação de formação de profissionais da química. ${ }^{16-20}$ 


\section{REAÇÃO DE ENAMINONAS E ACETILACETONA COM CLORETO DE 4-NITROFENILDIAZÔNIO}

Para exemplificar a participação de nucleófilos alifáticos no acoplamento azo, duas enaminonas derivadas da acetilacetona $\mathbf{1}$ foram sintetizadas, a 4-amino-pent-3-en-2-ona 2 e a 4-(fenilamino)-pent-3en-2-ona $\mathbf{3}$, esta última como descrito por Andrade e colaboradores, ${ }^{21}$ onde tanto o reator de micro-ondas doméstico quanto o dedicado para laboratório foram empregados, de forma que esta etapa se adaptou à realidade da infraestrutura do laboratório de ensino. Assim, as enaminonas $\mathbf{3}$ e $\mathbf{2}$ derivadas da anilina e da amônia, respectivamente, foram reagidas com o cloreto de 4-nitrofenildiazônio $\mathbf{5}$, preparado in situ, adaptando-se o procedimento descrito por Kascheres e Oliveira para a reação entre diazoquinonas e enaminonas. ${ }^{22} \mathrm{~A}$ escolha do sal de diazônio foi norteada por vários aspectos. A para-nitroanilina 4 , precursora do cloreto de 4-nitrofenildiazônio 5, é objeto de experimentos de graduação, sendo o produto final da rota clássica anilina/ acetanilida/4-nitroacetanilida/4-nitroanilina. ${ }^{1-5}$ Dessa forma, o produto final desta rota passa a ser também empregado em mais uma aula experimental, e a anilina utilizada na síntese da enaminona $\mathbf{3}$ é o precursor comum na síntese dos dois reagentes do acoplamento azo. Adicionalmente, a presença do grupo nitro nos azocompostos 6 e 7 confere-lhes maior cristalinidade, em função da possibilidade de formação de pontes de hidrogênio intermolecular, o que pode ser explorado para ampliar o escopo da aula aqui proposta, introduzindo os graduandos nas técnicas de obtenção de monocristal adequado para a determinação da estrutura por difração de raios X, como descrito abaixo.

Na síntese de $\mathbf{6}$ adaptou-se o procedimento do acoplamento azo descrito, avaliando-se a necessidade da etapa final de neutralização. ${ }^{22}$ A supressão desta etapa parece não comprometer o rendimento, pois quando 6 foi preparado e o isolamento feito imediatamente, o rendimento foi de $46 \%$, comparável ao do procedimento com neutralização $(51 \%)$. Como a diminuição de resíduos é um dos princípios da química verde, a não neutralização é aqui recomendada, Esquema 2. Para comparar a reatividade dos nucleófilos alifáticos no acoplamento azo e os aspectos estruturais dos corantes obtidos, a acetilacetona $\mathbf{1}$ foi empregada frente ao mesmo sal de diazônio $\mathbf{5}$, Esquema $3 .{ }^{23}$ Os rendimentos obtidos nos dois procedimentos (com e sem neutralização na etapa final) para o azocompostos $\mathbf{8}$ foram também comparáveis entre si e na mesma ordem de grandeza das reações com enaminona 3, Esquema 3.

A estratégia para o emprego das sínteses aqui descritas depende da duração da aula e do número de estudantes da turma. Para cursos de 4-6 h, é possível para uma só equipe a realização das duas preparações de corantes derivados das substâncias 1 e 3. Alternativamente, para cursos de 2-4h de duração, o instrutor pode dividir as sínteses entre as equipes e comparar os resultados, ficando esta decisão associada à infraestrutura laboratorial e à duração da aula, sendo possíveis várias combinações para atender aos diversos cursos. É importante destacar que a síntese da enaminona 3 é significativamente mais rápida que a de 2, pois a primeira pode ser feita empregando-se aquecimento por micro-ondas imediatamente antes do seu uso na reação seguinte do acoplamento azo, enquanto a síntese da enaminona $\mathbf{2}$ inicia numa aula e é finalizada na seguinte. Dessa forma, a realização da síntese do nucleófilo 2 só recompensa se associada ao experimento de difração de raios $\mathrm{X}$ da azoenaminona 7 correspondente.

Fotos coloridas (Figura S1), espectros de absorção na região do infravermelho e de ressonância magnética nuclear dos compostos 6-8 estão apresentados no Material Suplementar (Figuras S2 a S12). Os espectros de RMN de ${ }^{1} \mathrm{H}$ são particularmente interessantes, pois é possível detectar o conhecido equilíbrio tautomérico entre as formas azo e hidrazo dos compostos 6 e 7, Figura S3, bem como o equilíbrio

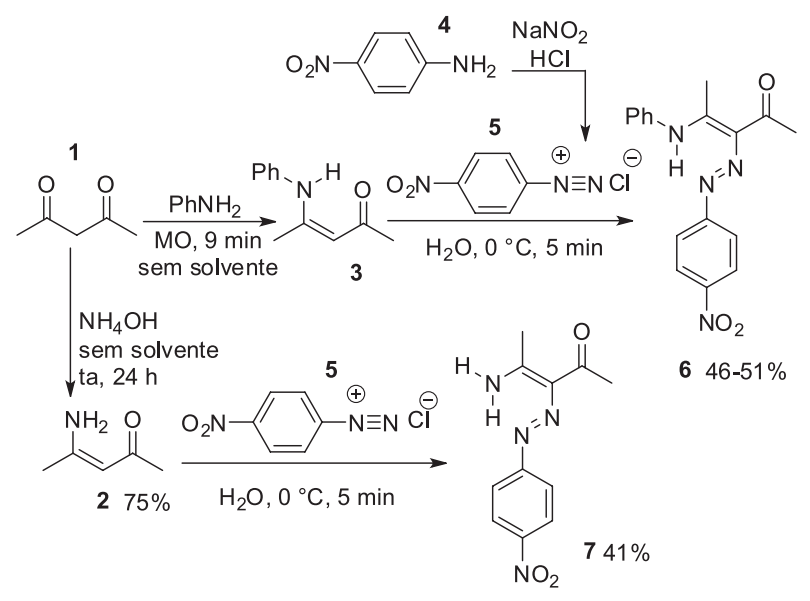

Esquema 2. Reações de acoplamento azo com enaminonas

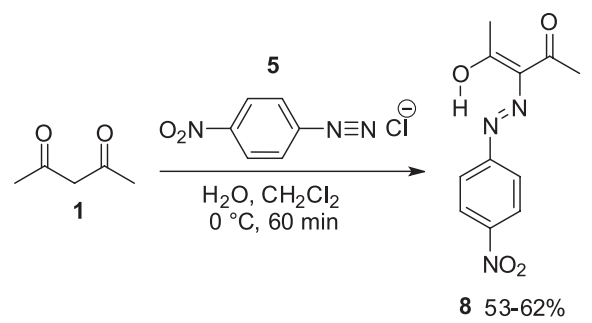

Esquema 3. Reação de acoplamento azo com acetilacetona

configuracional indicado no Esquema $4 .^{24,25}$ Todavia, não se pode determinar qual o isômero predomina por estas medidas. Entretanto, é possível fazer esta avaliação no estado sólido através da análise estrutural por difração de raios X. ${ }^{24,25}$ Interessantemente, o espectro de RMN de ${ }^{1} \mathrm{H}$ do produto $\mathbf{8}$ do acoplamento azo da acetilacetona apresenta apenas um isômero, sendo isolada a forma hidrazona. ${ }^{23}$ A observação dos referidos equilíbrios no azocomposto 7 pode ser discutido com os estudantes, sendo uma rica fonte de revisão dos conhecimentos de estereoquímica, Esquema 4. Por sua vez, a detecção de apenas um isômero para o azocomposto 8 (Figura S3) proporciona a comparação da natureza doadora de elétron do nitrogênio em $\mathbf{6}$ e $\mathbf{7}$ quando comparado ao oxigênio em $\mathbf{8}$, e a influência das forças relativas das pontes de hidrogênio intramoleculares nestes compostos $(\mathrm{N}-\mathrm{H} \cdots \mathrm{N}$ para as azoenaminonas 6 e 7 versus $\mathrm{O}-\mathrm{H} \cdots \mathrm{N}$ em 8$)$.

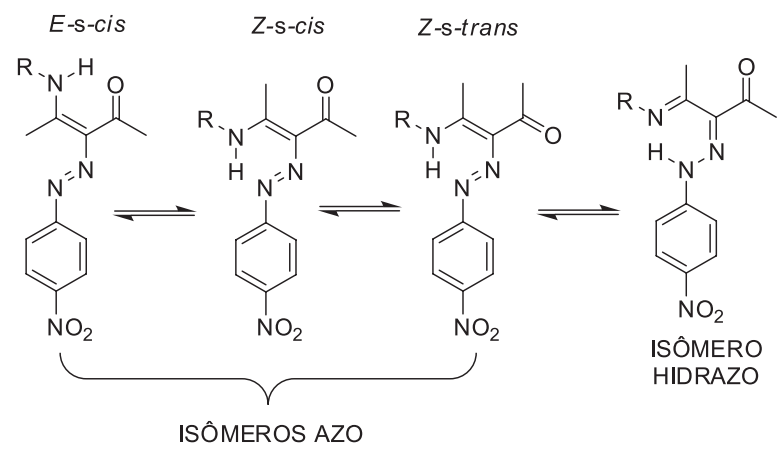

Esquema 4. Exemplos de formas isoméricas das azoenaminonas

\section{ESTRUTURA CRISTALINA DA AZOENAMINONA 7}

As azoenaminonas são conhecidas por formarem monocristais adequados para a determinação estrutural por difração de raios X.24-29 Em particular, a azoenaminona 7 forma monocristais com extrema facilidade e, por este aspecto, foi aqui selecionada para ser sintetizada e sua estrutura cristalina reinvestigada. 
A determinação da estrutura cristalina da azoenaminona 7 já foi descrita por Šimůnek e colaboradores ${ }^{24}$ Outras estruturas cristalinas de azoenaminonas também já foram descritas por outros pesquisadores, sendo um tema de interesse continuado. ${ }^{25-29}$ Para a reinvestigação da estrutura tridimensional de $\mathbf{7}$ aqui proposta, nas instituições onde o difratômetro de raios X está disponível, a estratégia da aula compreende a síntese da enaminona 2 numa primeira aula, a finalização na aula seguinte e o imediato acoplamento azo e a obtenção do monocristal no final do experimento, cabendo ao professor selecionar o cristal obtido pelos estudantes, que é então encaminhado ao setor de cristalografia para análise. Na aula seguinte, normalmente na semana consecutiva, os dados cristalográficos são apresentados e discutidos. A Figura 1 apresenta representações da estrutura 7 obtida em um desses experimentos. Caso o cristal inicialmente obtido não tenha qualidade para o estudo de difração por raios $\mathrm{X}$, o emprego de uma das técnicas de crescimento de monocristal fornece material adequado. ${ }^{10}$ Todavia, vale ressaltar que das diversas vezes que a azoenaminona 7 foi preparada em nosso laboratório, sempre foi possível a obtenção de monocristal, mesmo quando sintetizado por estudante ainda inexperiente.
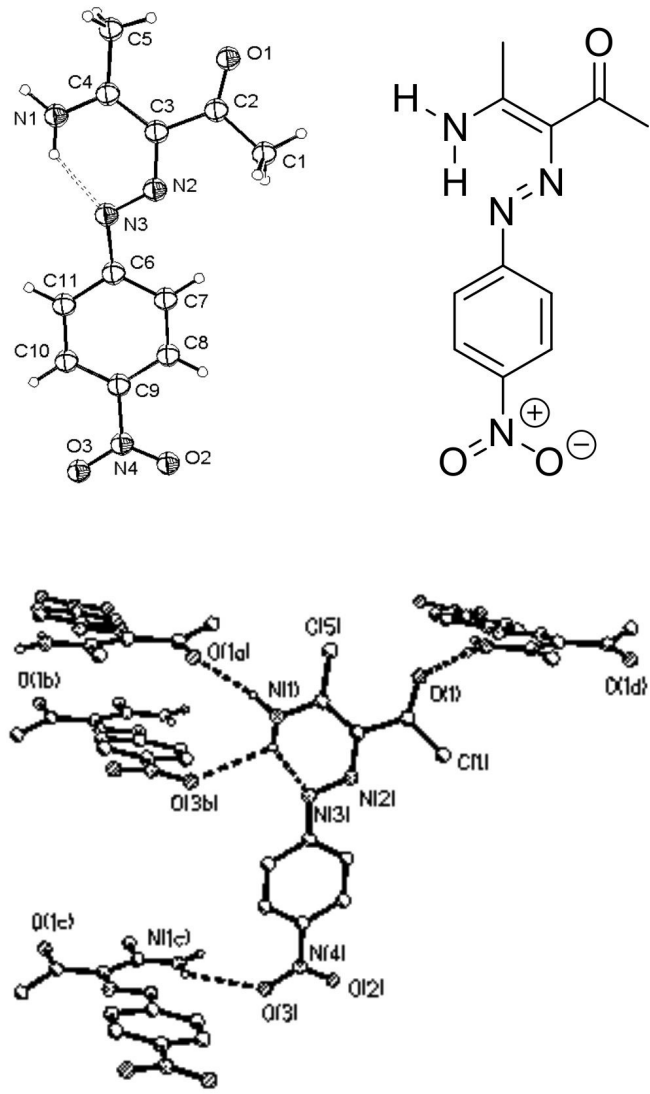

Figura 1. Duas visões da estrutura tridimensional da azoenaminona 7 por difração de raios $X$ com as pontes de hidrogênio intramolecular (acima) $e$ intermolecular (abaixo). Elipsoides são mostrados com nível de probabilidade de $30 \%$

Com a estrutura resolvida (Tabelas S1 a S4) é possível comparar a qualidade do cristal e dos dados cristalográficos obtidos com os descritos na literatura. ${ }^{24}$ Vários aspectos podem ser discutidos, como a diferença entre sólidos amorfos e cristalinos, o fenômeno de crescimento de cristal, os princípios da técnica de difração de raios $\mathrm{X}$, a natureza das forças inter e intramoleculares que governam o empacotamento cristalino, explicitadas na formação de pontes de hidrogênios e nos contatos próximos, a unidade assimétrica, enfim, um conjunto de variáveis pode ser abordado em função da familiaridade do instrutor com a cristalografia. Mesmo para os instrutores iniciantes na técnica, a realização do experimento aqui proposto pode motivar a aproximação entre o químico sintético e o cristalógrafo. $\mathrm{O}$ experimento integrado aqui proposto é passível de ser realizado também em instituições que não dispõem do difratômetro de raios $\mathrm{X}$, desde que se estabeleça colaboração com outra que o possua, como é o caso do IQ-UFBA e o IF-UFG.

Para o aproveitamento pleno do estudo cristalográfico, recomenda-se o envio do arquivo "CIF" aos estudantes, que podem instalar o programa Mercury disponível gratuitamente na internet. ${ }^{30} \mathrm{Com}$ este programa os próprios estudantes podem visualizar diversas projeções da estrutura cristalina e avaliar uma série de parâmetros estruturais tais como distâncias e ângulos de ligação, interações intra e intermoleculares, e o empacotamento cristalino da célula unitária. ${ }^{31}$

\section{CONCLUSÃO}

As enaminonas $\mathbf{2}$ e $\mathbf{3}$ e acetilacetona $\mathbf{1}$ são nucleófilos alifáticos reativos frente ao cloreto de 4-nitrofenildiazônio $\mathbf{5}$, formando azocompostos em rendimentos adequados para aulas de graduação. As sínteses das enaminonas são realizadas sem solvente e/ou empregando radiação de micro-ondas, de forma que a aula proposta é passível de aplicação imediata como parte integrante de disciplinas experimentais de química orgânica, pois os procedimentos são rápidos, simples, de fácil execução e empregam reagentes comuns de laboratórios de ensino experimental. Adicionalmente, a execução do experimento proporciona ao estudante discutir aspectos da química de sais de diazônios, de métodos atuais de promover reações químicas empregando micro-ondas e de Química Verde, que são aspectos importantes para a formação contemporânea de profissionais da química. Em adição, a execução da parte do estudo estrutural de monocristal de substâncias orgânicas por cristalografia de raios X passa a ser um diferencial na formação dos futuros profissionais da Química. ${ }^{32}$

\section{MATERIAL SUPLEMENTAR}

Arquivo PDF contendo fotos coloridas dos sólidos, espectros na região do infravermelho, espectros de $\mathrm{RMN}$ de ${ }^{1} \mathrm{H}$ e ${ }^{13} \mathrm{C}$, dados cristalográficos para compostos selecionados e o arquivo CIF da azoenaminona 7 encontram-se disponíveis gratuitamente em http:// quimicanova.sbq.org.br.

\section{PARTE EXPERIMENTAL}

Os pontos de fusão foram determinados em um aparelho de placa aquecida Microquímica MQAPF 301 e não foram corrigidos. $\mathrm{O}$ espectros na região do infravermelho foram obtidos na forma de discos de $\mathrm{KBr}$ em um aparelho SHIMADZU IR Affinity-1 ou no aparelho FT-IR Bomem MB100. Os espectros de RMN foram obtidos num aparelho Varian Gemini 300, e os deslocamentos químicos estão descritos em unidades de ppm a partir da referência (TMS). O forno de micro-ondas doméstico empregado foi da marca Electrolux, modelo MEF33. Os dados da cristalografia de raios X foram coletados a temperatura ambiente em um difratômetro Nonius CAD-4. ${ }^{33}$ A acetilacetona e a anilina são líquidos incolores, e foram destiladas antes do uso quando intensamente amarelas, ou empregadas como adquiridas se apenas levemente amareladas, não havendo significativa variação nos rendimentos.

\section{Síntese da enaminona 2}

Em um balão contendo $5 \mathrm{~mL}$ de acetilacetona $1 \mathrm{em}$ banho de gelo e com agitação magnética gotejar, empregando um funil de adição, 
$5 \mathrm{~mL}$ de $\mathrm{NH}_{4} \mathrm{OH}$. Ao término do gotejamento, retirar o banho de gelo e deixar reagir por 24 horas à temperatura ambiente. Adicionar 30 $\mathrm{mL}$ de solução saturada de cloreto de sódio e verter o conteúdo do balão para um funil de separação e extrair com 3 porções de $20 \mathrm{~mL}$ de diclorometano. Reunir a fase orgânica e secar com $\mathrm{MgSO}_{4}$, filtrar e eliminar o solvente no evaporador rotatório, o que fornece 3,65 g de um óleo amarelo, rendimento $75 \%$. A enaminona 2 assim obtida é utilizada na reação posterior sem purificação. Uma amostra analítica pode ser obtida por destilação a vácuo, $\mathrm{RMN}$ de ${ }^{1} \mathrm{H}\left(\mathrm{CDCl}_{3}\right): 1,91$ $(3 \mathrm{H}, \mathrm{s}), 2,02(3 \mathrm{H}, \mathrm{s}), 5,02(1 \mathrm{H}, \mathrm{s}) . \mathrm{RMN} \mathrm{de}{ }^{13} \mathrm{C}\left(\mathrm{CDCl}_{3}\right): 22,2\left(\mathrm{CH}_{3}\right)$; 29,0 $\left(\mathrm{CH}_{3}\right) ; 95,7(\mathrm{CH}) ; 161,34(\mathrm{C}) ; 196,7(\mathrm{C})$.

\section{Procedimento para a síntese da azoenaminona 7}

Em um balão de fundo redondo adicionar 1,38 g (10,0 mmol) da enaminona 2 e $0,69 \mathrm{~g}(5 \mathrm{mmol})$ de $p$-nitroanilina e $10 \mathrm{~mL}$ de água destilada. Acrescentar, com vigorosa agitação magnética e em banho de gelo, $3 \mathrm{~mL}$ de solução de $\mathrm{HCl} 6 \mathrm{~mol} \mathrm{~L}^{-1}$. Adicionar gota-a-gota uma solução previamente gelada contendo $15 \mathrm{~mL}$ de água destilada e $0,86 \mathrm{~g}(12,5 \mathrm{mmol})$ de nitrito de sódio $\left(\mathrm{NaNO}_{3}\right)$. Acrescentar 28 $\mathrm{mg}$ de uréia. Após esta etapa, neutralizar até pH 5-6 adicionando pequenas porções de $\mathrm{Na}_{2} \mathrm{CO}_{3}$ sólido. Após 5 minutos, adicionar lentamente a solução gelada previamente preparada com 1,0 g (10,0 mmol) da enaminona 2 (sintetizada no procedimento anterior) em $20 \mathrm{~mL}$ de diclorometano. O sólido vermelho que se forma é coletado por filtração e seco ao ar, fornecendo $0,92 \mathrm{~g}$ de 7 . Verter o filtrado para um funil de separação e extrair com $15 \mathrm{~mL}$ de diclorometano, separar a fase orgânica e secar com $\mathrm{MgSO}_{4}$ e filtrar. Adicionar éter de petróleo à solução e resfriar em freezer $\left(-25^{\circ} \mathrm{C}\right)$. Decantar a solução e secar o sólido vermelho adicional que se forma, o que fornece 0,11 g, totalizando $1,13 \mathrm{~g}$ de 7 (41\% de rendimento). PF $193,4-194,5^{\circ} \mathrm{C}$. IV (KBr): 3448, 3190, 1636, 1584, 1514, 1313, $805 \mathrm{~cm}^{-1}$. RMN de ${ }^{1} \mathrm{H}\left(\mathrm{CDCl}_{3}\right): 2,57(3 \mathrm{H}, \mathrm{s}) ; 2,60(3 \mathrm{H}, \mathrm{s}) ; 7,63(2 \mathrm{H}, \mathrm{d}, \mathrm{J}=8,7 \mathrm{~Hz}) ; 8.29$ $(2 \mathrm{H}, \mathrm{d}, \mathrm{J}=9 \mathrm{~Hz}) ; 14,32$ (sl).

\section{Síntese da enaminona $3 \mathrm{em}$ forno de micro-ondas doméstico}

Em um erlenmeyer de $125 \mathrm{~mL}$ adicionar $0,9 \mathrm{~mL}(10 \mathrm{mmol}) \mathrm{de}$ anilina e 1,0 mL (10 mmol) de acetilacetona. Colocar um funil de colo curto no erlenmeyer (para evitar projeções durante o aquecimento) e introduzir no aparelho de micro-ondas doméstico contendo, no centro da cavidade do forno, um béquer de $200 \mathrm{~mL}$ com $125 \mathrm{~mL}$ de água à temperatura ambiente. Programar o micro-ondas para aquecer na potência de $100 \mathrm{~W}$ por 3 minutos. Executar mais dois ciclos de aquecimento, deixando o forno desligado por um minuto entre cada ciclo e trocando a água do béquer entre os intervalos. O tempo total de aquecimento é 9 minutos. Análise por CCD tendo como eluente uma mistura de hexano e acetato de etila na proporção de 4:1 e revelação no UV ou iodo indica apenas uma mancha na placa. A enaminona 3 assim obtida é um óleo amarelo e é utilizado nas reações posteriores sem purificação, e seu espectro de infravermelho encontra-se na Figura 1S. Preparar uma solução com toda a massa obtida de $\mathbf{3}$ em 10 $\mathrm{mL}$ de diclorometano e resfriar em banho de gelo para ser utilizada na reação de diazotação abaixo.

\section{Procedimento com neutralização para a síntese da azoenaminona 6}

Em um balão de fundo redondo adicionar 690,7 mg (5 mmol) de p-nitroanilina e $5 \mathrm{~mL}$ de água. Acrescentar, com vigorosa agitação magnética e em banho de gelo, $3 \mathrm{~mL}$ de solução de $\mathrm{HCl} 6$ mol.L $\mathrm{L}^{-1}$. Adicionar gota-a-gota uma solução previamente gelada contendo $7 \mathrm{~mL}$ de água destilada e $347,1 \mathrm{mg}(6 \mathrm{mmol})$ de nitrito de sódio
$\left(\mathrm{NaNO}_{2}\right)$. Acrescentar $10 \mathrm{mg}$ de uréia. Após esta etapa, neutralizar até pH 5-6 adicionando pequenas porções de $\mathrm{Na}_{2} \mathrm{CO}_{3}$ sólido. Após 5 minutos, adicionar lentamente a solução gelada previamente preparada da enaminona 3 (sintetizada no procedimento anterior). Após 40 minutos, verter o conteúdo do balão para um funil de separação e extrair com 3 porções de $10 \mathrm{~mL}$ de diclorometano. Reunir a fase orgânica e secar com $\mathrm{MgSO}_{4}$, filtrar e eliminar o solvente no evaporador rotatório. O resíduo é recristalizado em acetona, e o sólido laranja que se forma é filtrado e seco, o que fornece $830 \mathrm{mg}$ de $\mathbf{6}$, $51 \%$ de rendimento. PF 146,3-148, $1^{\circ} \mathrm{C}$. IV (KBr) 3419, 1672, 1592 , $1514,1312,1165,1109,849,749,698 \mathrm{~cm}^{-1}$.

Se o sobrenadante da recristalização for deixado evaporar na bancada ocorre a precipitação de um sólido bege escuro, caracterizado como sendo o $p$-nitrofenol, subproduto da reação, PF 111,2-113,4 ${ }^{\circ} \mathrm{C}$. IV (KBr) 3275, 1609, 1596, 1511, 1329, 1250, 1168, 843, 748, $684 \mathrm{~cm}^{-1}$.

\section{Procedimento sem neutralização para a síntese da azoenaminona 6}

Em um balão de fundo redondo adicionar 1,38 g (10 mmol) de p-nitroanilina e $10 \mathrm{~mL}$ de água. Acrescentar, com vigorosa agitação magnética e em banho de gelo, $6 \mathrm{~mL}$ de $\mathrm{HCl} 6 \mathrm{~mol} \mathrm{~L}{ }^{-1}$. Adicionar gota-a-gota uma solução previamente gelada contendo $14 \mathrm{~mL}$ de água destilada e 0,83 g (12 mmol) de nitrito de sódio $\left(\mathrm{NaNO}_{2}\right)$. Acrescentar $20 \mathrm{mg}$ de uréia. Após 5 minutos, adicionar lentamente a solução gelada previamente preparada da enaminona $\mathbf{3}$ (sintetizada no procedimento anterior). Após 40 minutos, verter o conteúdo do balão para um funil de separação e extrair com 3 porções de $20 \mathrm{~mL}$ de diclorometano. Reunir a fase orgânica e secar com $\mathrm{MgSO}_{4}$, filtrar e eliminar o solvente no evaporador rotatório. O resíduo é recristalizado em acetona e o sólido laranja que se forma é filtrado e seco, o que fornece $1,48 \mathrm{~g}$ de $\mathbf{6}, 46 \%$ de rendimento. PF $145,5-148,4{ }^{\circ} \mathrm{C}$. IV (KBr) 3457, 1672, 1592, 1515, 1337, 1165, 849, 749, $698 \mathrm{~cm}^{-1}$.

Se sobrenadante da recristalização for deixado evaporar na bancada ocorre precipitação de um sólido bege escuro, caracterizado como sendo o $p$-nitrofenol, subproduto da reação, $\mathrm{PF} 110-112^{\circ} \mathrm{C}$. IV (KBr) 3275, 1609, 1596, 1511, 1329, 1250, 1168, 843, 748, $684 \mathrm{~cm}^{-1}$.

\section{Procedimento com neutralização para a síntese do azocomposto 8 derivado da acetilacetona}

Em um balão de fundo redondo adicionar $690,9 \mathrm{mg}$ ( $5 \mathrm{mmol}) \mathrm{de}$ p-nitroanilina e $10 \mathrm{~mL}$ de água. Acrescentar, com vigorosa agitação magnética e em banho de gelo, $3 \mathrm{~mL}$ de solução de $\mathrm{HCl} 6 \mathrm{~mol} \mathrm{~L}{ }^{-1}$. Adicionar gota-a-gota uma solução previamente gelada contendo 7 $\mathrm{mL}$ de água destilada $347,9 \mathrm{mg}(6 \mathrm{mmol})$ de nitrito de sódio $\left(\mathrm{NaNO}_{2}\right)$. Acrescentar 10 mg de uréia. Após esta etapa, neutralizar até pH 5-6 e adicionando pequenas porções de $\mathrm{Na}_{2} \mathrm{CO}_{3}$ sólido. Após 5 minutos, adicionar lentamente a solução gelada previamente preparada de 0,5 $\mathrm{mL}$ ( $5 \mathrm{mmol}$ ) de acetilacetona $\mathbf{1} \mathrm{em} 10 \mathrm{~mL}$ de diclorometano. Após 50 minutos, verter o conteúdo do balão para um funil de separação e extrair com 3 porções de $10 \mathrm{~mL}$ de diclorometano. Reunir a fase orgânica e secar com $\mathrm{MgSO}_{4}$, filtrar e eliminar o solvente no evaporador rotatório. O resíduo é recristalizado em acetona, e o sólido amarelo em forma de agulhas é filtrado e seco, o que fornece $766 \mathrm{mg}$ de 8, 62\% de rendimento. PF 223,6-225, ${ }^{\circ} \mathrm{C}$. IV (KBr) 3454, 3119, $1670,1605,1506,1334,1162,851,744,610 \mathrm{~cm}^{-1}$.

\section{Procedimento sem neutralização para a síntese do azocomposto 8 derivado da acetilacetona}

Em um balão de fundo redondo adicionar $1,38 \mathrm{~g}(10 \mathrm{mmol}) \mathrm{de}$ 
p-nitroanilina e $10 \mathrm{~mL}$ de água. Acrescentar, com vigorosa agitação magnética e em banho de gelo, $6 \mathrm{~mL}$ de $\mathrm{HCl} 6 \mathrm{~mol} \mathrm{~L}^{-1}$. Adicionar gota-a-gota uma solução previamente gelada contendo $14 \mathrm{~mL}$ de água destilada $0,83 \mathrm{~g}(12 \mathrm{mmol})$ de nitrito de sódio $\left(\mathrm{NaNO}_{2}\right)$. Acrescentar $20 \mathrm{mg}$ de uréia. Após 5 minutos, adicionar lentamente a solução gelada previamente preparada de $1 \mathrm{~mL}(10 \mathrm{mmol})$ de acetilacetona 1 em $15 \mathrm{~mL}$ de diclorometano. Após 50 minutos, verter o conteúdo do balão para um funil de separação e extrair com 3 porções de 20 $\mathrm{mL}$ de diclorometano. Reunir a fase orgânica e secar com $\mathrm{MgSO}_{4}$, filtrar e eliminar o solvente no evaporador rotatório. O resíduo é recristalizado em acetona, e o sólido amarelo em forma de agulhas é filtrado e seco, o que fornece $1,31 \mathrm{~g}$ de $\mathbf{8}, 53 \%$ de rendimento. PF $220,6-222,7^{\circ} \mathrm{C}$. IV (KBr) 3472, 1670, 1649, 1597, 1517, 1334, 1212, $1162,1110,851,744,610 \mathrm{~cm}^{-1}$.

\section{Cristalografia}

A estrutura foi resolvida com métodos diretos e refinada anisotropicamente usando o pacote de programas SHELXS-97 e SHELXL-97, ${ }^{33,34}$ respectivamente. As Tabelas S1 a S4 do Material Suplementar descrevem parâmetros estruturais selecionados.

\section{AGRADECIMENTO}

Os autores agradecem o suporte financeiro do Conselho Nacional de Desenvolvimento Científico e Tecnológico - CNPq, Coordenação de Aperfeiçoamento de Pessoal de Nível Superior - CAPES, e Fundação de Amparo à Pesquisa do Estado da Bahia - FAPESB. Também agradecemos à CAPES pela bolsa de mestrado de V. Rocha, ao CNPq pela bolsa de doutorado de e M. F. Marques e pela bolsa de produtividade em pesquisa de S. Cunha.

\section{REFERÊNCIAS}

1. Soares, B. G.; Souza, N. A.; Pires, D. X.; Química Orgânica: Teoria e Técnicas de Preparação, Purificação e Identificação de Compostos Orgânicos, Ed. Guanabara S.A.: Rio de Janeiro, 1988.

2. Furniss, B. S.; Hannaford, A. J.; Smith, P. W. G.; Tactchell, A. R.; Vogel's Textbook of Practical Organic Chemistry, $5^{\text {th }}$ ed., Longman Scientific \& Technical: Singapore, 1989.

3. Pavia, D. L.; Lampman, G. M.; Kriz, G. S.; Engel, R. G.; Introduction to Organic Laboratory Techniques: a Small Scale Approach, Saunders College Publishing: Philadelphia, 1998.

4. Tietze, L.-F., Eicher, T.; Reactions and Syntheses in the Organic Chemistry Laboratory, University Science Books: Mill Valley, California, 1989.

5. Fieser, L. F.; Experiments in Organic Chemistry, $3^{\text {rd }}$ ed., D. C. Heath and Company: Boston, 1955.

6. Taylor, J. G.; Moro, A. V.; Correia, C. R. D.; Eur. J. Org. Chem. 2011, 1403.

7. Cunha, S.; Santos Filho, R. F.; Riatto, V. B.; Dourado, G. A. A.; Quim. Nova 2013, 36, 190.

8. Cunha, S.; de Santana, L. L. B.; Quim. Nova 2012, 35, 642.

9. Cunha, S.; Lustosa, D. M.; Conceição, N. D.; Fascio, M.; Magalhães, V.; Quim. Nova 2012, 35, 638.
10. Cunha, S.; Quim Nova 2008, 31, 906

11. Cunha, S.; Beretta, M.; Fascio, M.; Santos, A. O.; Rodrigues Jr, M. T.; Bastos, R. M.; Quim. Nova 2005, 28, 364.

12. Cunha, S.; Lião, L. M.; Bonfim, R. R.; Bastos, R. M.; Monteiro A. P. M.; Alencar, K. S.; Quim. Nova 2003, 26, 425

13. Cunha, S.; Quim Nova 2003, 26, 948.

14. Bragg, W. L.; Bragg, W. H.; Proc. R. Soc. London, Ser. A 1913, 89, 277. Para comemorar o centenário do Prêmio Nobel de Max Laue (Willian Lawrence Bragg e seu pai Willian Henry Bragg foram laureados em 1915), em função da descoberta que os raios X sofrem difração ao passarem por um cristal, o ano de 2014 foi escolhido como o Ano Internacional da Cristalografia pela Assembleia Geral das Nações Unidas, e uma série de eventos já estão em curso, e vários materiais didáticos estão sendo disponibilizados gratuitamente em: http://www. iycr2014.org/contribute/educational-materials, acessada em 12/01/2013.

15. Thomas, J. M.; Angew. Chem. Int. Ed. 2012, 51, 12946; Santin Filho, O.; Quim. Nova 1995, 18, 574.

16. Crundwell, G.; Phan, J.; Kantardjieff, K. A.; J. Chem. Educ. 1999, 76, 1242 .

17. Hoggard, P. E.; J. Chem. Educ. 2002, 79, 420.

18. Geremia, S.; Demitri, N.; J. Chem. Educ. 2005, 82, 460.

19. Szalay, P. S.; Zeller, M.; Hunter, A. D.; J. Chem. Educ. 2005, 82, 1555.

20. Guzei, I. A.; Hill, N. J.; Zakai, U. I.; J. Chem. Educ. 2010, 87, 1257.

21. Andrade, C. K. Z.; Fátima, A.; Barreto, A. S.; Silva, W. A.; Arkivoc 2008, 12, 226.

22. Figueiredo, L. J. O.; Kascheres, C.; J. Org. Chem. 1997, 62, 1164.

23. Lyčka, A.; Šnobl, D.; Collect. Czech. Chem. Commun. 1981, 46, 892.

24. Šimůnek, P.; Bertolasi, V.; Macháček, V.; J. Mol. Struct. 2002, 642, 41.

25. Šimůnek, P.; Svobodová, M.; Bertolasi, V.; Pretto, L.; Lyčka, A.; Macháček, V.; New J. Chem. 2007, 31, 429.

26. Kettmann, V.; Lokaj, J.; Šimůnek, P.; Macháček, V.; Acta Crystallogr., Sect. C: Cryst. Struct. Commun. 2001, 57, 737.

27. Šimůnek, P.; Macháček, V.; Dyes Pigm. 2010, 86, 197.

28. Rodrigues, B. L.; Gambardella, M. T. P.; Figueiredo, L. J. O.; Kascheres, C.; Acta Crystallogr., Sect. C: Cryst. Struct. Commun. 1996, 52, 705.

29. Vencato, I.; Cunha, S.; Rocha, V.; Rocha, Z. N.; Lariucci, C.; Acta Crystallogr, Sect. E: Struct. Rep. Online 2004, 60, o1704.

30. http://www.ccdc.cam.ac.uk/free_services/mercury/downloads/, acessada em 03/01/2013.

31. Professores e estudantes que desejarem analisar previamente os dados cristalográficos do azocomposto 7 através do programa Mercury podem utilizar o arquivo CIF disponível no material suplementar.

32. Para exemplos de outras abordagens de experimentos para a graduação envolvendo difração de raios X, ver: Müller, R. A.; Batres, E. J. Q.; Quim. Nova 1995, 18, 382; Zarbin, A. J. G.; Davanzo, C. U.; Quim. Nova 1995, 18, 494; Galembeck, A.; Gushiken, Y.; Quim. Nova 1996, 19, 200; Santana, G. P.; Ramos, A. M.; Fabris, J. D.; Quim. Nova 2008, 31, 430; Oliveira, H. F. N.; Trinca, R. B.; Gushiken, Y.; Quim. Nova 2009, 32, 1346.

33. Enraf-Nonius; CAD4/PC Version 1.2. Enraf-Nonius, Delft, Holanda, 1993.

34. Sheldrick, G. M.; SHELXS97; Program for the Solution of Crystal Structures; University of Göttingen, Alemanha, 1997; Sheldrick, G. M.; SHELXL97; Program for the Refinement of Crystal Structures; University of Göttingen, Alemanha, 1997. 